\title{
Ab initio Studies of Magnetism in the Iron Chalcogenides FeTe and FeSe
}

\author{
Motoaki Hirayama* ${ }^{1}$ Takahiro Misawa, ${ }^{2}$ Takashi Miyake ${ }^{3}$ and Masatoshi Imada ${ }^{2}$ \\ ${ }^{1}$ Department of Physics, Tokyo Institute of Technology, Ookayama, Meguro-ku, Tokyo 152-8551, Japan \\ ${ }^{2}$ Department of Applied Physics, University of Tokyo, 7-3-1 Hongo, Bunkyo-ku, Tokyo 113-8656, Japan \\ ${ }^{3}$ Nanomaterials Research Institute, AIST, Tsukuba, Ibaraki 305-8568, Japan
}

\begin{abstract}
The iron chalcogenides FeTe and FeSe belong to the family of iron-based superconductors. We study the magnetism in these compounds in the normal state using the $a b$ initio downfolding scheme developed for strongly correlated electron systems. In deriving ab initio low-energy effective models, we employ the constrained $G W$ method to eliminate the double counting of electron correlations originating from the exchange correlations already taken into account in the density functional theory. By solving the derived $a b$ initio effective models, we reveal that the elimination of the double counting is important in reproducing the bicollinear antiferromagnetic order in FeTe, as is observed in experiments. We also show that the elimination of the double counting induces a unique degeneracy of several magnetic orders in FeSe, which may explain the absence of the magnetic ordering. We discuss the relationship between the degeneracy and the recently found puzzling phenomena in FeSe as well as the magnetic ordering found under pressure.
\end{abstract}

After the discovery of iron-based superconductors, many families of the iron-based superconductors have been found. ${ }^{1-3)}$ Among them, iron chalcogenides such as FeTe and FeSe (11-type) are the simplest iron-based superconductors, which have no blocking insulating layer. Despite the simple lattice structure of the 11-type compounds, many characteristic behaviors have been observed in them, which have attracted much interest. For example, FeTe shows an exceptional bicollinear antiferromagnetic (AFB) order with an ordered moment of $\left.\sim 2.0-2.25 \mu_{\mathrm{B}}, 4,5\right)$ although most of the iron-based superconductors show a stripe antiferromagnetic (AFS) order. Although the AFB order itself is reproduced by several calculations based on the density functional theory (DFT) ${ }^{6-9)}$ the microscopic origin of the AFB order and the roles of electron correlations are not fully clarified yet.

In FeSe, although calculation based on the DFT predicts that the AFS order is the ground state, ${ }^{8)}$ no clear signature of magnetic order is observed in experiments, and the superconducting phase appears below $T_{c} \sim 8$ $K^{10)}$ Note that a structural phase transition without magnetic order occurs at $T_{s} \sim 90 \mathrm{~K}$. The absence of the magnetic order despite its large local moment ${ }^{11)}$ remains a puzzle in FeSe. Recent experimental result ${ }^{12-14)}$ supports the notion that FeSe has extremely small Fermi surfaces and that the resultant superconductivity is located in the Bose-Einstein-condensate (BEC) region. The origin of these exotic phenomena also remains a puzzle in FeSe. Furthermore, it is reported that a single layer of FeSe on $\mathrm{SrTiO}_{3}$ shows high-temperature superconductivity, whose $T_{c}$ is higher than $50 \mathrm{~K}^{15)}$ Thus, clarifying these remarkable behaviors in iron chalcogenides is important for understanding the nature of high-temperature superconductivity in iron-based superconductors.

Many theoretical and experimental studies indicate

*E-mail: hirayama@stat.phys.titech.ac.jp that the 11-type compounds are located in the strongcoupling region. ${ }^{11-14,16-20)}$ In particular, in recent experimental studies of a single crystal of FeSe, ${ }^{12-14)}$ it has been shown that the experimental Fermi surfaces cannot be explained by the conventional DFT calculations. In addition, an $a b$ initio evaluation of the interaction parameters suggests that the 11-type compounds have relatively stronger correlations than other iron-based superconductors such as $\mathrm{BaFe}_{2} \mathrm{As}_{2}$ and LaFeAsO. ${ }^{17)}$ In the strong-coupling region, it is necessary to consider the effects of electron correlations beyond the conventional DFT. Even when the strong electron correlation is taken into account, the above recently studied Fermi surface does not appear consistent with the calculated Fermi surface. ${ }^{18,21)}$ This result is a puzzling aspect of FeSe.

A hybrid method ( $a b$ initio downfolding scheme) ${ }^{22)}$ of the DFT and precise model calculation is a promising way of treating electron correlations beyond the standard DFT: In this scheme, we first obtain the global band structure based on the DFT and evaluate the interaction parameters in the low-energy effective model by constrained random-phase approximation (cRPA). ${ }^{23,24)}$ Then, we solve the low-energy effective model using precise low-energy solvers such as the many-variable variational Monte Carlo (mVMC) method. ${ }^{25)}$ Several applications to real materials show the validity and accuracy of this scheme ${ }^{26-28)}$. However, in this scheme, there is a fundamental problem in that the electron correlations are doubly counted, i.e., the electron correlations are considered in both the band calculations and the model calculations. Although this double counting is assumed to be small in the conventional downfolding scheme, in the strong-coupling regime where iron chalcogenides are expected to be located, the elimination of double counting may become important to correctly reproduce electronic structures.

In this Letter, by employing the state-of-the-art $a b$ initio method, we clarify the magnetic properties in iron 


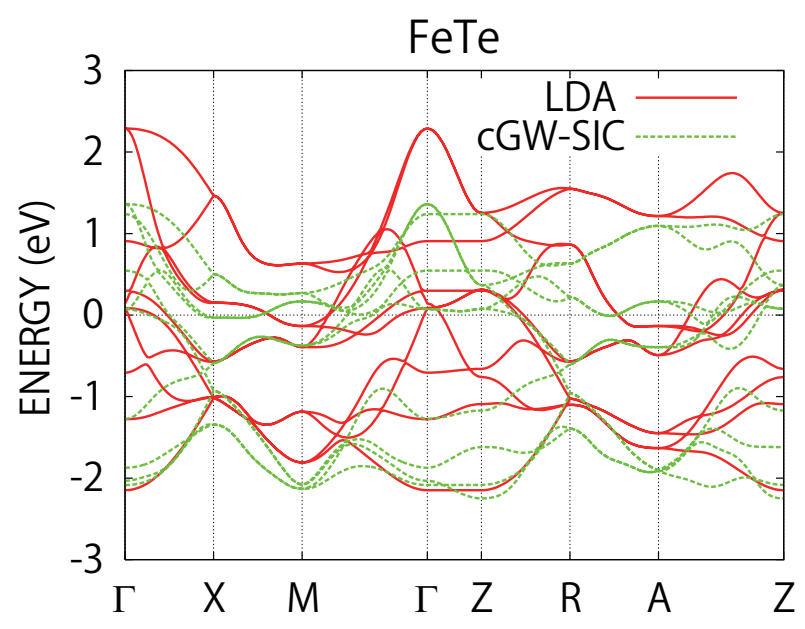

Fig. 1. (Color online) Electronic band structures of the Fe $3 d$ MLWFs of FeTe in the LDA [(red) solid line] and the cGW-SIC [(green) dashed line]. The Fermi energy is set to zero.

chalcogenides. We properly eliminate the double counting of the electron correlations and derive the low-energy effective models in an $a b$ initio way. ${ }^{29)}$ Then, we solve the low-energy effective models using the mVMC. As a result, we show that the elimination of the double counting plays a crucial role in stabilizing the AFB in FeTe. We also solve the low-energy effective models for FeSe in addition to $\mathrm{FeTe}$, and find that several magnetic orders are energetically nearly degenerate. This degeneracy may result in the absence of the magnetic order in FeSe under competitions and frustrations emerging in the region close to the phase boundaries.

Our low-energy effective models for iron-based superconductors are given as follows:

$$
\begin{gathered}
\mathcal{H}_{\mathrm{eff}}^{\mathrm{X}}=\sum_{i j} \sum_{m n \sigma} t_{m n \sigma}^{\mathrm{X}}\left(\boldsymbol{R}_{i}-\boldsymbol{R}_{j}\right) d_{i n \sigma}^{\dagger} d_{j m \sigma}+\frac{1}{2} \sum_{i j} \sum_{m n \sigma \rho} \\
\left\{U_{m n \sigma \rho}\left(\boldsymbol{R}_{i}-\boldsymbol{R}_{j}\right) d_{i n \sigma}^{\dagger} d_{j m \rho}^{\dagger} d_{j m \rho} d_{i n \sigma}+J_{m n \sigma \rho}\left(\boldsymbol{R}_{i}-\boldsymbol{R}_{j}\right)\right. \\
\left.\left(d_{i n \sigma}^{\dagger} d_{j m \rho}^{\dagger} d_{i n \rho} d_{j m \sigma}+d_{i n \sigma}^{\dagger} d_{i n \rho}^{\dagger} d_{j m \rho} d_{j m \sigma}\right)\right\}, \quad(1) \\
t_{m n \sigma}^{\mathrm{X}}(\boldsymbol{R})=\left\langle\phi_{m \mathbf{0}}\left|\mathcal{H}^{\mathrm{X}}\right| \phi_{n \boldsymbol{R}}\right\rangle,
\end{gathered}
$$

where $t^{\mathrm{X}}$ represents the transfer integral of the maximally localized Wannier functions (MLWFs). ${ }^{30,31)}$ Here, $\mathrm{X}$ denotes the methods of deriving the one-body part of the Hamiltonian, i.e., $\mathrm{X}=\mathrm{LDA}$ (cGW-SIC) means that the local density approximation (constrained GW-selfinteraction correction) is used for evaluating transfer integrals. We will show details of the cGW-SIC later. The corresponding band structures near the Fermi level are shown in Fig. 1. Here, $d_{i n \sigma}^{\dagger}\left(d_{i n \sigma}\right)$ is a creation (annihilation) operator of an electron with spin $\sigma$ in the $n$th MLWF having strong Fe $3 d$ characters (with $n=1-5$ being the orbital index) centered at $\boldsymbol{R}_{i} \cdot{ }^{17)}$ In this work, we take the $\mathrm{X}$ - and $\mathrm{Y}$-axes for the MLWFs index along the Fe-Te/Se directions. Note that the $12 \times 12 \times 6 \boldsymbol{k}$-mesh is employed in the LDA calculation, and the $6 \times 6 \times 3$ $\boldsymbol{k}$-mesh is employed in the cRPA and cGW-SIC calculations. The interaction parameters $U$ and $J$ denote the Coulomb interactions and exchange interactions, respectively. These interaction parameters are calculated by the cRPA $^{22,23)}$ and dimensional downfolding. ${ }^{27,32,33)}$ For details of the interaction parameters and hopping parameters, see Ref. 34. The off-site interactions $(i \neq j)$ are omitted in this calculation because they are less than one-quarter of the on-site interaction parameter. Double counting in the Hartree term is subtracted as we have mentioned in our previous paper. ${ }^{26)}$

For the one-body part of the Hamiltonian, in addition to the standard LDA calculations, the constrained GW (cGW) scheme was employed, ${ }^{29,35)}$ which can eliminate the double counting of the self-energy originating from the exchange-correlation energy. In this scheme, we first subtract the exchange-correlation energy in the LDA calculations and replace it with the $G W$ self-energy calculated by excluding the low-energy contribution, since the low-energy part of the self-energy will be considered later when the obtained model is solved. In this scheme, since we do not consider the low-energy part of the $G W$ self-energy in deriving the low-energy effective model, the double counting of exchange correlations does not occur.

The cGW self-energy does not include the selfinteraction (SI) originating from the low-energy degree of freedom, which should be canceled out by the oppositesign SI in the Hartree term. In the nondegenerate multiband systems, the imbalance of the SI exerts crucial effects on the occupation number of each orbital and the magnetism. We therefore introduce the self-interaction correction (SIC) originating from the low-energy degrees of freedom $U^{\text {on-site }} n_{\mathrm{LDA}}^{L} / 2$ into the cGW scheme, where $U^{\text {on-site }}$ is the on-site effective interaction and $n_{\mathrm{LDA}}^{L} / 2$ is the occupation number of the low-energy degrees of freedom of the up or down spin in the LDA. The one-body part with the SIC is given by

$$
\mathcal{H}^{\mathrm{cGW}-\mathrm{SIC}}=\mathcal{H}^{\mathrm{cGW}}-Z^{\mathrm{cGW}} U^{\mathrm{on}-\text { site }} \frac{n_{\mathrm{LDA}}^{L}}{2},
$$

where $\mathcal{H}^{\mathrm{cGW}}$ is the static one-body Hamiltonian in the cGW. ${ }^{29)}$ The renormalization factor $Z^{\mathrm{cGW}}$ is needed to renormalize the frequency-dependent part of the interaction of the effective model. ${ }^{29)}$

Figure 1 shows the band structures of FeTe in the LDA and cGW-SIC, which correspond to the one-body part of the low-energy effective model in Eq. (1). We note that the band structure of the cGW-SIC model for the noninteracting case is a virtual one, which cannot be directly observed in experiments. It is expected that correct band structures are reproduced after solving the low-energy effective models. The main difference between the LDA and cGW-SIC models is the on-site potential of the $3 Z^{2}-R^{2}$ orbital. In the cGW-SIC model, the on-site potential of the $3 Z^{2}-R^{2}$ orbital decreases and the occupation of the $3 Z^{2}-R^{2}$ orbital increases. As we will show later, the stability of the magnetic order largely depends on the on-site potential of the $3 Z^{2}-R^{2}$ orbital.

To solve the low-energy effective models, we employ the $\mathrm{mVMC}$, which can treat the electron correlations se- 


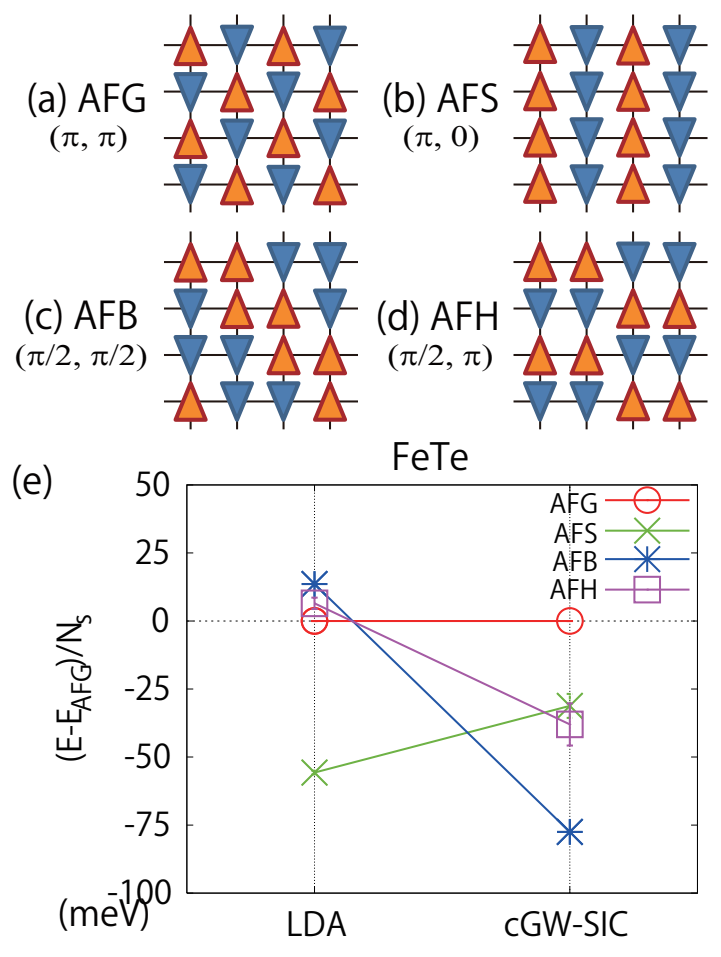

Fig. 2. (Color online) AF patterns calculated in this work: (a) AFG, (b) AFB, (c) AFS, and (d) AFH. (e) Groundstate energy per site of $\mathrm{AF}$ in $\mathrm{mVMC}$ extrapolated to the thermodynamic limit for FeTe with $t^{\mathrm{LDA}}$ and with $t^{\mathrm{cGW}-\mathrm{SIC}}$ (in $\mathrm{meV}$ ). The energy $E$ is extrapolated to the thermodynamic limit $E_{\infty}$ by employing the scaling $E=E_{\infty}+a N_{\mathrm{s}}^{-3 / 228,36)}$ with a constant $a$ for the system size $N_{\mathrm{s}}$.

riously. ${ }^{25)}$ Our variational wavefunction is defined as

$$
|\psi\rangle=\mathcal{P}_{\mathrm{J}} \mathcal{P}_{\mathrm{G}} \mathcal{L}^{S=0}\left|\psi_{\text {pair }}\right\rangle
$$

where $\left|\psi_{\text {pair }}\right\rangle$ is a generalized pairing wave function, and $\mathcal{L}^{S=0}, \mathcal{P}_{\mathrm{G}}$, and $\mathcal{P}_{\mathrm{J}}$ are the total spin quantum-number projection, Gutzwiller factors, and Jastrow factors, respectively. ${ }^{25,26)}$ For more computational details of ironbased superconductors, readers are referred to Ref. 26 .

In the $\mathrm{mVMC}$, we examine the stability of four possible AF orders, i.e., G-type AF state (AFG), AFS, AFB, and half-collinear AF state (AFH), whose schematic pictures are shown in Figs. 2(a)-2(d), respectively. We mainly show the results of $N_{\mathrm{s}}=8 \times 8$ sites with periodic boundary conditions; the systems with $4 \times 4$ and $4 \times 8$ sites are also calculated for the extrapolation to the thermodynamic limit.

By solving the low-energy effective models, we show how the elimination of double counting affects the stability of magnetic orders in FeTe. As shown in Figs. 2(a)$2(\mathrm{~d})$, we examine four different magnetic orders, which are potential candidates of the order. In the LDA model, we find that the AFS is the most stable magnetically ordered phase [Fig. 2(e)]. In contrast, the AFB phase becomes the ground state in the cGW-SIC calculation that properly eliminates the double counting of the selfenergy. This result indicates the importance of the elimination of the double counting in the strong-coupling regime.

In Table I, we show the orbital resolved occupation
Table I. Occupation numbers of the Wannier functions of the LDA and cGW-SIC models in the mVMC, where the sum of the occupancy is 6 . Error bars are negligible $\left(\leq 10^{-3}\right)$.

\begin{tabular}{c|ccccc}
\hline \hline FeSe (AFH) & $X Y$ & $Y Z$ & $3 Z^{2}-R^{2}$ & $Z X$ & $X^{2}-Y^{2}$ \\
\hline LDA & 1.14 & 1.36 & 1.02 & 1.32 & 1.16 \\
cGW-SIC & 1.27 & 1.04 & 1.64 & 1.04 & 1.01 \\
\hline FeTe (AFB) & $X Y$ & $Y Z$ & $3 Z^{2}-R^{2}$ & $Z X$ & $X^{2}-Y^{2}$ \\
\hline LDA & 1.40 & 1.12 & 1.12 & 1.31 & 1.05 \\
cGW-SIC & 1.44 & 1.05 & 1.42 & 1.07 & 1.02 \\
\hline \hline
\end{tabular}

number $n_{\nu}$ for both the LDA model and the cGW-SIC model. Compared with that in the LDA model, the occupation number of the $Y Z / Z X$ orbitals becomes close to half filling $\left(n_{Y Z / Z X} \sim 1\right)$ in addition to the $X^{2}-Y^{2}$ orbital. This is caused by the lowering of the on-site potential of the $3 Z^{2}-R^{2}$ orbital in the cGW-SIC model. The localization of the $X^{2}-Y^{2}$ orbital is frequently referred to as the orbital selective Mottness in the literature. ${ }^{18,26,27,37-39)}$ In addition, our result indicates that the $Y Z / Z X$ orbitals also show orbital-selective Mott insulating behavior in FeTe as well as in FeSe. The orbital selective Mott insulating behaviors are evidenced by small double occupancies $d_{m}=\left\langle n_{m \uparrow} n_{m \downarrow}\right\rangle=0.1$ for $m=$ $Y Z / Z X$ and $=0.04$ for $m=X^{2}-Y^{2}$ and small orbitalresolved charge compressibilities $\chi_{\mathrm{cm}}=d n_{m} / d \mu<0.001$ $(\mathrm{meV})^{-1}$ for $m=Y Z / Z X$ and $X^{2}-Y^{2}$ in FeTe (AFB) $(\mu=d E / d N$ is the uniform chemical potential applied to all the orbitals, where $E$ is the total energy and $N$ is the total number of electrons). ${ }^{40)}$ Because the occupancies of the $Y Z / Z X$ orbitals are slightly different from half filling, the $Y Z / Z X$ orbitals can be regarded as doped orbital-selective Mott insulators in a strict sense, where the excess carriers from half filling make the system metallic. ${ }^{41)}$ This additional localization may be the origin of the characteristic behavior observed in iron chalcogenides. Note that a similar localization is observed in previous calculations for iron chalcogenides. ${ }^{20,39)}$

Here, we examine the microscopic origin of the bicollinear magnetic order in FeTe. In previous calculations based on the $\mathrm{DFT}^{6,7)}$, although it was suggested that the origin of the AFB order is attributed to large farther-neighbor magnetic interactions such as the thirdneighbor magnetic interaction $J_{3}$, it is unclear what induces the large farther-neighbor magnetic interactions. One possible origin of such large farther-neighbor interactions is the superexchange interaction that is induced by farther-neighbor hoppings. To examine the effects of farther-neighbor hoppings, we artificially cut off fartherneighbor hoppings. As shown in Fig. 3(a), we find that the AFB becomes the ground state regardless of the range of such hoppings. The robustness of the AFB indicates that the superexchange interactions induced by the farther-neighbor hoppings play minor roles in stabilizing the AFB order.

Another possible origin of the farther-neighbor magnetic interactions is Ruderman-Kittel-Kasuya-Yosida (RKKY) interactions, which are mainly induced by the 


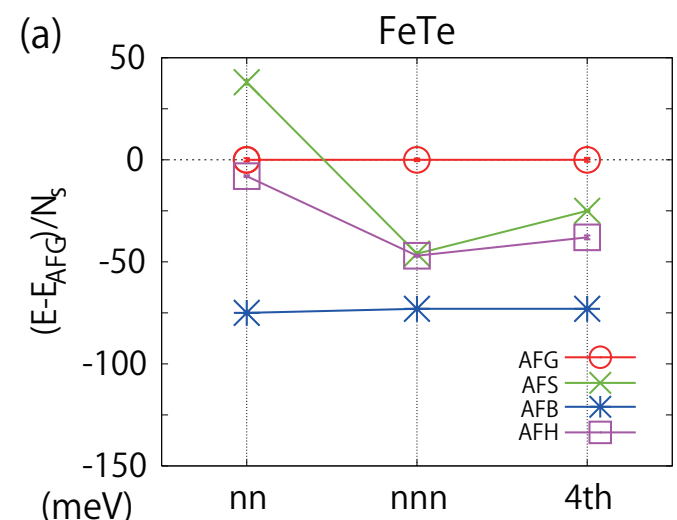

(b)

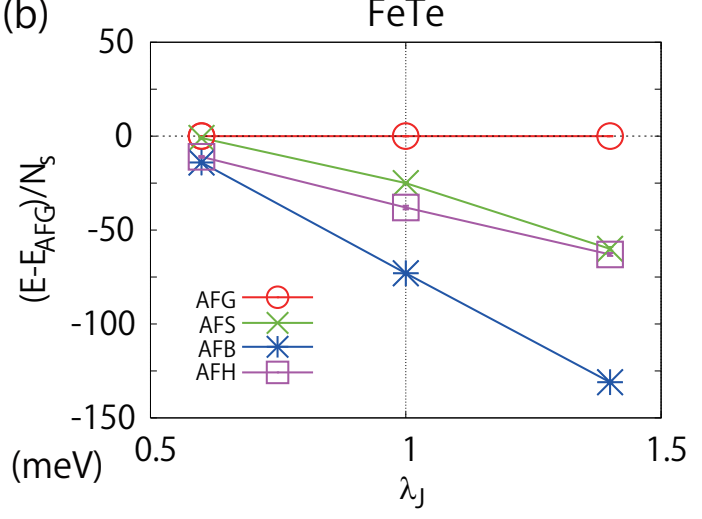

Fig. 3. (Color online) Ground-state energy per site of AF in the mVMC for FeTe with the cGW-SIC parameter (a) as a function of cutoff length of $t$ and (b) as a function of ratio for $J, \lambda_{J}\left(J \rightarrow \lambda_{J} \cdot J\right)$ (in $\mathrm{meV}$ ). The nearest-neighbor, next-nearest-neighbor, and 4thneighbor hoppings are included in "nn", "nnn", and "4th" calculations, respectively. Energy is measured from that of the AFG. The system size is $N_{\mathrm{s}}=8 \times 8$.

Hund's rule coupling. To examine the roles of the Hund's rule coupling as well as of the RKKY interactions, we artificially change the magnitude of the Hund's rule coupling by introducing the scaling parameter $\lambda_{J}$, where $\lambda_{J}$ scales on-site exchange interactions as $J \rightarrow \lambda_{J} J$. Figure $3(\mathrm{~b})$ shows the ground-state energy per site of several AF orders in FeTe as a function of $\lambda_{J}$. The AFB becomes more stable as $J$ becomes larger. This result indicates that the RKKY-type interactions play key roles in stabilizing the AFB order. Since scattering decreases with the magnetic ordering, the resistivity decreases well below the magnetic transition temperature ${ }^{41)}$ particularly along the $(\pi / 2,-\pi / 2)$ ferromagnetic line. ${ }^{42,43)}$ The structural transition $^{44)}$ would enhance the energy stabilization through the changes in transfer parameters. The couplings between itinerant electrons and localized spins resulting in the RKKY interactions were examined on phenomenological levels. ${ }^{45-47)}$

Now, we discuss the magnetic properties of FeSe. As in the case of FeTe, the AFS phase becomes the ground state in the LDA calculation for FeSe, as shown in Fig. 4. For FeSe, we only show the results of the largest system size $\left(N_{s}=8 \times 8\right)$, because the size dependence of the energy is not smooth in FeSe, while we estimate the thermodynamic limit to be not far from the result for $N_{s}=8 \times 8$. By eliminating the double counting of the self-energy, we find that the AFH phase has the lowest energy, which is consistent with previous ab initio calculations. ${ }^{48,49)}$ What is more characteristic of FeSe is that the AFH and AFB phases win the AFS state and they have nearly the same energy, i.e., the difference in the energy between the AFH and AFB is on the order of $10 \mathrm{meV}$ and the energies of the AFS and AFG phases are also close within $25 \mathrm{meV}$. Such degeneracies were not properly captured in the previous DFT-GGA studies ${ }^{48,49)}$ where the energies of the AFB and AFG phases are much higher. Because of the limitation of the system sizes and the scattered size dependence of energy for FeSe, the energy stability of FeSe has a large uncertainty with an energy scale of $10 \mathrm{meV}$. Thus, this degeneracy does not contradict the experimental results that the magnetic order is not observed despite its large local moment, since it is probable that the short-range order of the AFB and AFH (and AFS as well) coexist as a mixed or fluctuating phase, which does not allow the development of the long-range order above the superconducting transition temperature.

It is naively expected that the superposition of the degenerate magnetic orders will induce spin-glass-like states. However, the spin-glass-like states are not consistent with the existence of small but clear Fermi surfaces ${ }^{12,13)}$ In the magnetically degenerate region, it is plausible that an unconventional superconducting phase becomes stable. By examining the stability of the superconducting phase by extending the present analysis for the normal states, the origin of small Fermi surfaces and the resultant BEC-like superconductivity ${ }^{14)}$ will be clarified. Further theoretical investigation of such a possibility is left for future study. It is also interesting to determine the shapes of the Fermi surfaces from jumps in momentum distributions by performing the calculations for larger system sizes.

The energy of the AFG phase is relatively low in FeSe compared with that in FeTe. The reason why the energy of the AFG phase is low is that the occupation number of the $X Y$ orbital having a very weak frustration is also close to half filling (1.10) in addition to those of the $Y Z / Z X$ and $X^{2}-Y^{2}$ having stronger frustrations. Note that the AFG order is more stable if the unfrustrated orbitals are closer to half filling. This reduction of the $X Y$ filling originates from the remarkable decrease in the onsite potential of the $3 Z^{2}-R^{2}$ orbital in FeSe; thus, the filling of the other orbitals must decrease. However, the $Y Z / Z X$ and $X^{2}-Y^{2}$ orbitals are already pinned near half filling. This drives the filling of the $X Y$ orbital closer to half filling. Because the $X Y$ orbital partially resolves the frustration in comparison to FeTe and the energy of the AFG phase becomes lower, FeSe shows an accidental degeneracy of several phases. More precisely, when the three orbitals $\left(Y Z, Z X\right.$, and $\left.X^{2}-Y^{2}\right)$ could become close to half filling by an appropriate on-site potential, the AFS, AFB, and AFH phases may have similar energies. If the $X Y$ orbital in addition could become close to half filling, the AFG phase joins in this degeneracy.

As we will show later, the degeneracy may be lifted easily under pressure. The difference in the crystal field splitting from FeTe yields the unique energetic degener- 


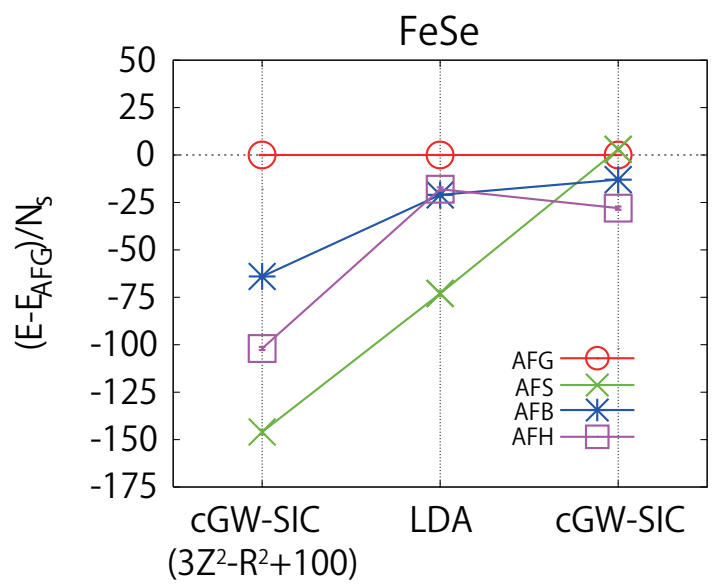

Fig. 4. (Color online) Ground-state energy per site of AF in the mVMC for FeSe with $t^{\mathrm{LDA}}$ and with $t^{\mathrm{cGW}-\mathrm{SIC}}$ (in meV). The left-hand data is obtained by using the cGW-SIC calculation, but the on-site potential of the $3 Z^{2}-R^{2}$ orbital is increased by 100 meV artificially. The system size is $N_{\mathrm{s}}=8 \times 8$.

acy of the AF orders in FeSe.

Lastly, we examine the robustness of the degeneracy in FeSe. By comparing the results of the LDA and cGWSIC models, it is speculated that the stability of the AF phases largely depends on the on-site potential of the $3 Z^{2}-R^{2}$ orbital in iron chalcogenides. To determine the roles of the on-site potential directly, we monitor the effect of the on-site potential of the $3 Z^{2}-R^{2}$ in the cGW-SIC model for FeSe. As a result, we find that the degeneracy is easily lifted and that the AFS becomes the ground state as shown in Fig. 4. This result can be understood by considering the fact that the occupation number of the $Y Z / Z X$ orbitals deviates from the half filling, and that only the $X^{2}-Y^{2}$ orbital, which stabilizes the AFS states through large next-nearest transfer, stays near half filling and becomes the sole driving force of the magnetism. This mechanism is hardly captured by weakcoupling theories. ${ }^{50)}$ Although not so easy, experimental control of the one-body potential of the $3 Z^{2}-R^{2}$ orbital will reveal the origin of the degeneracy in FeSe. The magnetic transition under pressure ${ }^{51,52)}$ would be related to the degeneracy of the AF phases. In the present ab initio study using the cGW-SIC model of FeSe under 4 GPa, such a degeneracy is lifted and it predicts that the AFH phase becomes stable, while the AFS phase is competing with it. ${ }^{53)}$

In this Letter, we have shown that the elimination of the double counting in the exchange-correlation energy is essential for reproducing the AFB order observed in FeTe. The stability of the AFB in FeTe is insensitive to farther-neighbor transfer but sensitive to the strength of the Hund's rule coupling. These results indicate that the nesting in Fermi surfaces and superexchange interactions play minor roles but that the RKKY interactions induced by the Hund's rule coupling play a key role in stabilizing the AFB order. We have also found that several magnetic orders are energetically degenerate in FeSe. This degeneracy is the possible origin of the absence of magnetic order in FeSe. It is shown that this degeneracy can be lifted by changing the chemical potential of the $3 Z^{2}-R^{2}$ orbital. Our analysis provides a firm theoretical basis for understanding the characteristic magnetism in iron chalcogenides and offers a clue to understanding other unique behaviors observed in the iron chalcogenides such as the possible high-temperature superconductivity in a single layer of FeSe. ${ }^{15)}$

We would like to thank Kazuma Nakamura for discussions on the dimensional downfolding method and Taichi Terashima for discussions on FeSe under pressure. This work has been supported by Grants-in-Aid for Scientific Research from the Ministry of Education, Culture, Sports, Science and Technology of Japan (MEXT) under grant numbers 22104010 and 22340090 . This work has also been financially supported by MEXT HPCI Strategic Programs for Innovative Research (SPIRE) and Computational Materials Science Initiative (CMSI). We also acknowledge $\mathrm{K}$ computer at RIKEN Advanced Institute for Computational Science (AICS) under grant numbers hp120043, hp120283, hp130007, hp140215, and hp150211.

1) K. Ishida, Y. Nakai, and H. Hosono, J. Phys. Soc. Jpn. 78, 062001 (2009).

2) G. R. Stewart, Rev. Mod. Phys. 83, 1589 (2011).

3) D. J. Scalapino, Rev. Mod. Phys. 84, 1383 (2012).

4) S. Li, C. de la Cruz, Q. Huang, Y. Chen, J. W. Lynn, J. Hu, Y.-L. Huang, F.-C. Hsu, K.-W. Yeh, M.-K. Wu, and P. Dai, Phys. Rev. B 79, 054503 (2009).

5) W. Bao, Y. Qiu, Q. Huang, M. A. Green, P. Zajdel, M. R. Fitzsimmons, M. Zhernenkov, S. Chang, M. Fang, B. Qian, E. K. Vehstedt, J. Yang, H. M. Pham, L. Spinu, and Z. Q. Mao, Phys. Rev. Lett. 102, 247001 (2009).

6) F. Ma, W. Ji, J. Hu, Z.-Y. Lu, and T. Xiang, Phys. Rev. Lett. 102, 177003 (2009).

7) C.-Y. Moon and H. J. Choi, Phys. Rev. Lett. 104, 057003 (2010).

8) J. Kumar, S. Auluck, P. Ahluwalia, and V. Awana, Supercond. Sci. Technol. 25, 095002 (2012).

9) M.-C. Ding, H.-Q. Lin, and Y.-Z. Zhang, Phys. Rev. B 87, 125129 (2013).

10) F.-C. Hsu, J.-Y. Luo, K.-W. Yeh, T.-K. Chen, T.-W. Huang, P. M. Wu, Y.-C. Lee, Y.-L. Huang, Y.-Y. Chu, D.-C. Yan, and M.-K. Wu, Proc. Natl. Acad. Sci. USA 105, 14262 (2008).

11) H. Gretarsson, A. Lupascu, J. Kim, D. Casa, T. Gog, W. Wu, S. R. Julian, Z. J. Xu, J. S. Wen, G. D. Gu, R. H. Yuan, Z. G. Chen, N.-L. Wang, S. Khim, K. H. Kim, M. Ishikado, I. Jarrige, S. Shamoto, J.-H. Chu, I. R. Fisher, and Y.-J. Kim, Phys. Rev. B 84, 100509 (R) (2011).

12) T. Terashima, N. Kikugawa, A. Kiswandhi, E.-S. Choi, J. S. Brooks, S. Kasahara, T. Watashige, H. Ikeda, T. Shibauchi, Y. Matsuda, T. Wolf, A. E. Böhmer, F. Hardy, C. Meingast, H. v. Löhneysen, M.-T. Suzuki, R. Arita, and S. Uji, Phys. Rev. B 90, 144517 (2014).

13) T. Shimojima, Y. Suzuki, T. Sonobe, A. Nakamura, M. Sakano, J. Omachi, K. Yoshioka, M. Kuwata-Gonokami, K. Ono, H. Kumigashira, A. E. Böhmer, F. Hardy, T. Wolf, C. Meingast, H. v. Löhneysen, H. Ikeda, and K. Ishizaka, Phys. Rev. B 90, 121111 (2014).

14) S. Kasahara, T. Watashige, T. Hanaguri, Y. Kohsaka, T. Yamashita, Y. Shimoyama, Y. Mizukami, R. Endo, H. Ikeda, K. Aoyama, T. Taichi, U. Shinya, W. Thomas, v. L. Hilbert, S. Takasada, and M. Yuji, Proc. Natl. Acad. Sci. USA 111, 16309 (2014).

15) W. Qing-Yan, L. Zhi, Z. Wen-Hao, Z. Zuo-Cheng, Z. Jin-Song, L. Wei, D. Hao, O. Yun-Bo, D. Peng, C. Kai, W. Jing, S. Can- 
Li, H. Ke, J. Jin-Feng, J. Shuai-Hua, W. Yayu, W. Lili, C. Xi, M. Xucun, and X. Qi-Kun, Chinese Physics Letters 29, $037402(2012)$.

16) K. Nakayama, T. Sato, P. Richard, T. Kawahara, Y. Sekiba, T. Qian, G. F. Chen, J. L. Luo, N. L. Wang, H. Ding, and T. Takahashi, Phys. Rev. Lett. 105, 197001 (2010).

17) T. Miyake, K. Nakamura, R. Arita, and M. Imada, J. Phys. Soc. Jpn. 79, 044705 (2010).

18) M. Aichhorn, S. Biermann, T. Miyake, A. Georges, and M. Imada, Phys. Rev. B 82, 064504 (2010).

19) Z. P. Yin, K. Haule, and G. Kotliar, Nat. Mater. 10, 932 (2011).

20) N. Lanatà, H. U. R. Strand, G. Giovannetti, B. Hellsing, L. de' Medici, and M. Capone, Phys. Rev. B 87, 045122 (2013).

21) J. M. Tomczak, M. van Schilfgaarde, and G. Kotliar, Phys. Rev. Lett. 109, 237010 (2012).

22) M. Imada and T. Miyake, J. Phys. Soc. Jpn. 79, 112001 (2010).

23) F. Aryasetiawan, M. Imada, A. Georges, G. Kotliar, S. Biermann, and A. I. Lichtenstein, Phys. Rev. B 70, 195104 (2004).

24) T. Miyake and F. Aryasetiawan, Phys. Rev. B 77, 085122 (2008).

25) D. Tahara and M. Imada, J. Phys. Soc. Jpn 77, 114701 (2008).

26) T. Misawa, K. Nakamura, and M. Imada, J. Phys. Soc. Jpn. 80, 023704 (2011).

27) T. Misawa, K. Nakamura, and M. Imada, Phys. Rev. Lett. 108, 177007 (2012).

28) H. Shinaoka, T. Misawa, K. Nakamura, and M. Imada, J. Phys. Soc. Jpn. 81, 034701 (2012).

29) M. Hirayama, T. Miyake, and M. Imada, Phys. Rev. B 87, 195144 (2013).

30) N. Marzari and D. Vanderbilt, Phys. Rev. B 56, 12847 (1997).

31) I. Souza, N. Marzari, and D. Vanderbilt, Phys. Rev. B 65, 035109 (2001)

32) K. Nakamura, Y. Yoshimoto, Y. Nohara, and M. Imada, J. Phys. Soc. Jpn. 79, 123708 (2010).

33) K. Nakamura, private communication.

34) See Supplemental Material for details of transfers and interaction parameters.

35) F. Aryasetiawan, J. M. Tomczak, T. Miyake, and R. Sakuma, Phys. Rev. Lett. 102, 176402 (2009).

36) D. A. Huse, Phys. Rev. B 37, 2380(R) (1988).

37) H. Ishida and A. Liebsch, Phys. Rev. B 81, 054513 (2010).

38) M. Greger, M. Kollar, and D. Vollhardt, Phys. Rev. Lett. 110 , 046403 (2013).

39) L. de' Medici, G. Giovannetti, and M. Capone, Phys. Rev. Lett. 112, 177001 (2014).

40) T. Misawa and M. Imada, Nat. Commun. 5, 5738 (2014).

41) Y. Mizuguchi and Y. Takano, J. Phys. Soc. Jpn. 79, 102001 (2010).

42) J. Jiang, C. He, Y. Zhang, M. Xu, Q. Q. Ge, Z. R. Ye, F. Chen, B. P. Xie, and D. L. Feng, Phys. Rev. B 88, 115130 (2013).

43) L. Liu, T. Mikami, M. Takahashi, S. Ishida, T. Kakeshita, K. Okazaki, A. Fujimori, and S. Uchida, Phys. Rev. B 91, 134502 (2015)

44) A. Martinelli, A. Palenzona, M. Tropeano, C. Ferdeghini, M. Putti, M. R. Cimberle, T. D. Nguyen, M. Affronte, and C. Ritter, Phys. Rev. B 81, 094115 (2010).

45) W.-G. Yin, C.-C. Lee, and W. Ku, Phys. Rev. Lett. 105, 107004 (2010).

46) A. Akbari, P. Thalmeier, and I. Eremin, New J. Phys. 15, 033034 (2013)

47) S. Ducatman, R. M. Fernandes, and N. B. Perkins, Phys. Rev. B 90, 165123 (2014).

48) H.-Y. Cao, S. Chen, H. Xiang, and X.-G. Gong, Phys. Rev. B 91, 020504(R) (2015).

49) J. K. Glasbrenner, I. I. Mazin, H. O. Jeschke, P. J. Hirschfeld, and R. Valenti. arXiv:1501.04946.

50) K. Suzuki, H. Usui, and K. Kuroki, Phys. Rev. B 84, 144514 (2011).

51) M. Bendele, A. Amato, K. Conder, M. Elender, H. Keller, H.H. Klauss, H. Luetkens, E. Pomjakushina, A. Raselli, and R. Khasanov, Phys. Rev. Lett. 104, 087003 (2010).

52) T. Terashima, N. Kikugawa, S. Kasahara, T.Watashige, T.
Shibauchi, Y. Matsuda, T. Wolf, A. E. Bohmer, F. Hardy, C. Meingast, H. v. Lohneysen, and S. Uji, J. Phys. Soc. Jpn. 84, 063701 (2015).

53) See Supplemental Material for details of the magnetism of FeSe under pressure. 


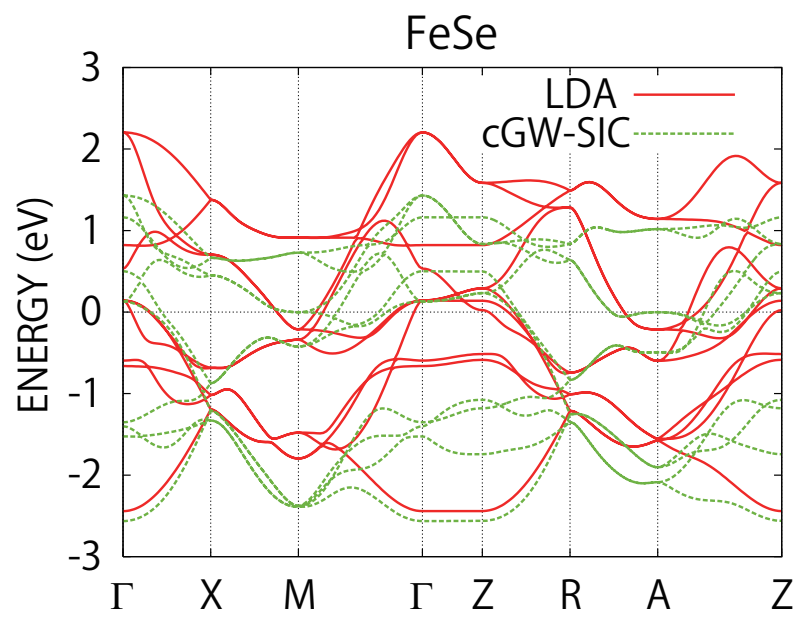

Fig. S 1. (Color online) Electronic band structures of the Fe $3 d$ MLWFs of FeSe in the LDA [(red) solid line] and the cGW-SIC [(green) dashed line]. The Fermi energy is set to zero.

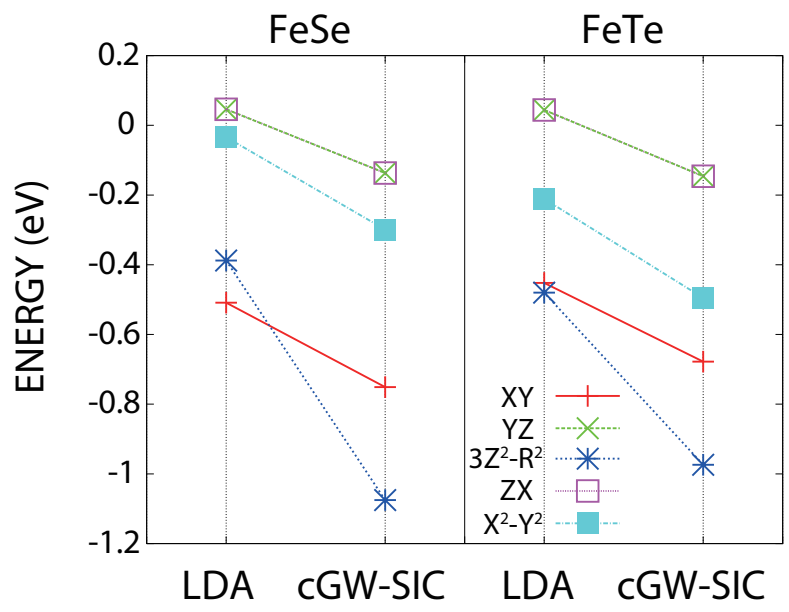

Fig. S 2. (Color online) On-site potential of Wannier orbitals for FeSe and FeTe obtained by the LDA and cGW-SIC calculations.

\section{Supplemental Materials}

\section{S.1 Values of Parameters of Low-Energy Effec- tive Model}

In this supplemental material, we show the values of the parameters of the $a b$ initio low-energy effective model. Table S I (II) is the three-dimensional effective interaction of the maximally localized Wannier functions (MLWFs) originating from the $\mathrm{Fe} 3 d$ orbitals of FeSe (FeTe). ${ }^{\mathrm{S} 1)}$ We obtain the two-dimensional effective interaction by uniformly subtracting $0.6(0.4) \mathrm{eV}$ from the three-dimensional effective interaction of $\mathrm{FeSe}(\mathrm{FeTe})$ following Refs. S2 and S3. Table S III is the transfer integral of the MLWFs of FeSe and FeTe obtained by the local-density-approximation (LDA) calculation. We also show the transfer integral of the MLWFs in the constrained GW with the self-interaction correction (cGWSIC) model in Table S IV. Figure S 1 is the corresponding band structure of FeSe. The on-site potential of each model is shown in Fig. S 2. The detail of the cGW method is explained in Refs. S1 and S4.

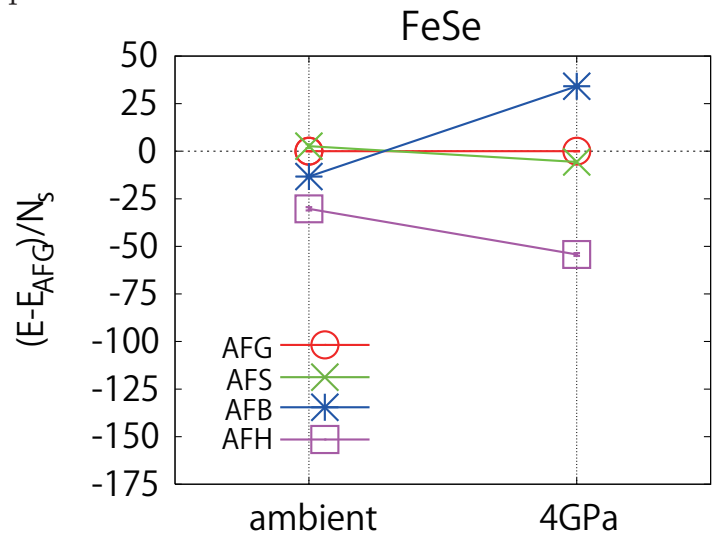

Fig. S 3. (Color online) Ground state energy per site of AF in the $\mathrm{mVMC}$ for FeSe at ambient pressure and at $4.0 \mathrm{GPa}$ with $t^{\mathrm{cGW}-\mathrm{SIC}}$ (in $\mathrm{meV}$ ). System size is $N_{\mathrm{s}}=8 \times 8$.

\section{S.2 Magnetism of FeSe under Pressure}

We calculate the magnetism of FeSe under pressure as well as that at ambient pressure. Figure S 3 shows the energy per site by the cGW-SIC calculation for FeSe at $4.0 \mathrm{GPa}$ in the experimental geometry. ${ }^{\mathrm{S} 5)}$ Although the AFS-type short-ranged fluctuations were observed $\left.{ }^{\mathrm{S} 6}, \mathrm{~S} 7\right)$ at ambient pressure, the unique energetic degeneracy at ambient pressure is lifted under pressure, and we predict that the AFH phase becomes slightly more stable than the AFS. The experimental verification would be desired.

[S1] M. Hirayama, T. Miyake, and M. Imada, Phys. Rev. B 87, 195144 (2013).

[S2] K. Nakamura, Y. Yoshimoto, Y. Nohara, and M. Imada, J. Phys. Soc. Jpn. 79, 123708 (2010).

[S3] K. Nakamura, private communication.

[S4] F. Aryasetiawan, J. M. Tomczak, T. Miyake, and R. Sakuma, Phys. Rev. Lett. 102, 176402 (2009).

[S5] S. Margadonna, Y. Takabayashi, Y. Ohishi, Y. Mizuguchi, Y. Takano, T. Kagayama, T. Nakagawa, M. Takata, and K. Prassides, Phys. Rev. B 80, 064506 (2009).

[S6] M. C. Rahn, R. A. Ewings, S. J. Sedlmaier, S. J. Clarke, and A. T. Boothroyd, Phys. Rev. B 91, 180501(R) (2015).

[S7] Q. Wang, Y. Shen, B. Pan, Y. Hao, M. Ma, F. Zhou, P. Steffens, K. Schmalzl, T. R. Forrest, M. Abdel-Hafiez, D. A. Chareev, A. N. Vasiliev, P. Bourges, Y. Sidis, H. Cao, J. Zhao, arXiv:1502.07544. 
Table S I. Bare and effective three-dimensional Coulomb interactions between two electrons for all the combinations of Fe $3 d$ orbitals in FeSe (in $\mathrm{eV}$ ). Here, $v$ and $J_{v}$ represent the bare on-site and exchange Coulomb interactions, respectively. The static limit of the effective on-site and exchange Coulomb interactions are denoted by $U$ and $J$, respectively.

\begin{tabular}{|c|c|c|c|c|c|c|c|c|c|c|}
\hline \multirow[t]{2}{*}{ FeSe } & \multicolumn{5}{|c|}{$v$} & \multicolumn{5}{|c|}{$U$} \\
\hline & $X Y$ & $Y Z$ & $3 Z^{2}-R^{2}$ & $Z X$ & $X^{2}-Y^{2}$ & $X Y$ & $Y Z$ & $3 Z^{2}-R^{2}$ & $Z X$ & $X^{2}-Y^{2}$ \\
\hline$X Y$ & 18.65 & 16.50 & 17.28 & 16.50 & 16.51 & 4.51 & 3.19 & 3.21 & 3.19 & 3.48 \\
\hline$Y Z$ & 16.50 & 16.97 & 17.05 & 15.70 & 15.30 & 3.19 & 4.11 & 3.53 & 3.02 & 2.98 \\
\hline $3 Z^{2}-R^{2}$ & 17.28 & 17.05 & 19.09 & 17.05 & 16.00 & 3.21 & 3.53 & 4.68 & 3.53 & 3.01 \\
\hline$Z X$ & 16.50 & 15.70 & 17.05 & 16.97 & 15.30 & 3.19 & 3.02 & 3.53 & 4.11 & 2.98 \\
\hline \multirow[t]{3}{*}{$X^{2}-Y^{2}$} & 16.51 & 15.30 & 16.00 & 15.30 & 15.88 & 3.48 & 2.98 & 3.01 & 2.98 & 3.77 \\
\hline & \multicolumn{5}{|c|}{$J_{v}$} & \multicolumn{5}{|c|}{$J$} \\
\hline & $X Y$ & $Y Z$ & $3 Z^{2}-R^{2}$ & $Z X$ & $X^{2}-Y^{2}$ & $X Y$ & $Y Z$ & $3 Z^{2}-R^{2}$ & $Z X$ & $X^{2}-Y^{2}$ \\
\hline$X Y$ & & 0.66 & 0.79 & 0.66 & 0.34 & & 0.57 & 0.69 & 0.57 & 0.32 \\
\hline$Y Z$ & 0.66 & & 0.46 & 0.56 & 0.61 & 0.57 & & 0.42 & 0.48 & 0.53 \\
\hline $3 Z^{2}-R^{2}$ & 0.79 & 0.46 & & 0.46 & 0.75 & 0.69 & 0.42 & & 0.42 & 0.62 \\
\hline$Z X$ & 0.58 & 0.56 & 0.46 & & 0.61 & 0.57 & 0.48 & 0.42 & & 0.53 \\
\hline$X^{2}-Y^{2}$ & 0.34 & 0.61 & 0.75 & 0.61 & & 0.32 & 0.53 & 0.62 & 0.53 & \\
\hline
\end{tabular}

Table S II. Bare and effective three-dimensional Coulomb interactions between two electrons for all the combinations of Fe $3 d$ orbitals in FeTe (in eV). Here, $v$ and $J_{v}$ represent the bare on-site and exchange Coulomb interactions, respectively. The static limit of the effective on-site and exchange Coulomb interactions are denoted by $U$ and $J$, respectively.

\begin{tabular}{|c|c|c|c|c|c|c|c|c|c|c|}
\hline \multirow[t]{2}{*}{$\mathrm{FeTe}$} & \multicolumn{5}{|c|}{$v$} & \multicolumn{5}{|c|}{$U$} \\
\hline & $X Y$ & $Y Z$ & $3 Z^{2}-R^{2}$ & $Z X$ & $X^{2}-Y^{2}$ & $X Y$ & $Y Z$ & $3 Z^{2}-R^{2}$ & $Z X$ & $X^{2}-Y^{2}$ \\
\hline$X Y$ & 17.08 & 15.05 & 16.20 & 15.05 & 16.26 & 3.46 & 2.30 & 2.36 & 2.30 & 2.80 \\
\hline$Y Z$ & 15.05 & 15.36 & 15.87 & 14.24 & 14.94 & 2.30 & 3.08 & 2.62 & 2.15 & 2.30 \\
\hline $3 Z^{2}-R^{2}$ & 16.20 & 15.87 & 18.25 & 15.87 & 16.08 & 2.36 & 2.62 & 3.73 & 2.62 & 2.35 \\
\hline$Z X$ & 15.05 & 14.24 & 15.87 & 15.36 & 14.94 & 2.30 & 2.15 & 2.62 & 3.08 & 2.30 \\
\hline \multirow[t]{3}{*}{$X^{2}-Y^{2}$} & 16.32 & 14.94 & 16.08 & 14.94 & 16.77 & 2.82 & 2.30 & 2.36 & 2.30 & 3.39 \\
\hline & \multicolumn{5}{|c|}{$J_{v}$} & \multicolumn{5}{|c|}{$J$} \\
\hline & $X Y$ & $Y Z$ & $3 Z^{2}-R^{2}$ & $Z X$ & $X^{2}-Y^{2}$ & $X Y$ & $Y Z$ & $3 Z^{2}-R^{2}$ & $Z X$ & $X^{2}-Y^{2}$ \\
\hline$X Y$ & & 0.59 & 0.73 & 0.59 & 0.33 & & 0.49 & 0.62 & 0.49 & 0.31 \\
\hline$Y Z$ & 0.59 & & 0.42 & 0.49 & 0.59 & 0.49 & & 0.37 & 0.40 & 0.49 \\
\hline $3 Z^{2}-R^{2}$ & 0.73 & 0.42 & & 0.42 & 0.74 & 0.62 & 0.37 & & 0.37 & 0.62 \\
\hline$Z X$ & 0.59 & 0.49 & 0.42 & & 0.59 & 0.49 & 0.40 & 0.37 & & 0.49 \\
\hline$X^{2}-Y^{2}$ & 0.33 & 0.59 & 0.74 & 0.59 & & 0.31 & 0.49 & 0.62 & 0.49 & \\
\hline
\end{tabular}


Table S III. Transfer integral for the $3 d$ orbitals of the Fe sites in the FeSe and FeTe, $t_{m n}^{\mathrm{LDA}}\left(R_{x}, R_{y}, R_{z}\right)$, where $t^{\mathrm{LDA}}$ is the expectation value of the Kohn-Sham Hamiltonian for the Wannier function : $t^{\mathrm{LDA}}=\left\langle\phi\left|\mathcal{H}^{\mathrm{LDA}}\right| \phi\right\rangle, m$ and $n$ denote symmetries of the $3 d$ orbitals, and the axis of $\left(R_{x}, R_{y}, R_{z}\right)$ is taken along the $\mathrm{Fe}-\mathrm{Se} / \mathrm{Te}$ directions. Units are given in meV.

\begin{tabular}{|c|c|c|c|c|c|c|c|c|c|c|}
\hline \multicolumn{11}{|l|}{ FeSe } \\
\hline$(m, n) \backslash \boldsymbol{R}$ & {$[0,0,0]$} & {$\left[\frac{1}{2},-\frac{1}{2}, 0\right]$} & {$[1,0,0]$} & {$[1,-1,0]$} & {$\left[\frac{3}{2},-\frac{1}{2}, 0\right]$} & {$\left[0,0, \frac{c}{a}\right]$} & {$\left[\frac{1}{2},-\frac{1}{2}, \frac{c}{a}\right]$} & $\sigma_{Y}$ & $I$ & $\sigma^{L}$ \\
\hline$(X Y, X Y)$ & -509 & -410 & -70 & -11 & 3 & -25 & 6 & + & + & + \\
\hline$(X Y, Y Z)$ & 0 & 273 & 131 & -9 & -6 & 0 & -9 & + & - & $-(1,4)$ \\
\hline$\left(X Y, 3 Z^{2}-R^{2}\right)$ & 0 & -347 & 0 & 22 & -8 & 0 & 11 & - & + & + \\
\hline$(X Y, Z X)$ & 0 & 273 & 0 & -9 & 18 & 0 & -3 & - & - & $-(1,2)$ \\
\hline$\left(X Y, X^{2}-Y^{2}\right)$ & 0 & 0 & 0 & 0 & -9 & 0 & -4 & - & + & - \\
\hline$(Y Z, Y Z)$ & 46 & 197 & 128 & -17 & -8 & 8 & 27 & + & + & $(4,4)$ \\
\hline$\left(Y Z, 3 Z^{2}-R^{2}\right)$ & 0 & -119 & 0 & 7 & 2 & 0 & 11 & - & - & $-(4,3)$ \\
\hline$(Y Z, Z X)$ & 0 & 127 & 0 & -23 & -19 & 0 & 12 & - & + & $(4,2)$ \\
\hline$\left(Y Z, X^{2}-Y^{2}\right)$ & 0 & 223 & 0 & 1 & -3 & 0 & 20 & - & - & $(4,5)$ \\
\hline$\left(3 Z^{2}-R^{2}, 3 Z^{2}-R^{2}\right)$ & -388 & -4 & -15 & -14 & -6 & -23 & -9 & + & + & + \\
\hline$\left(3 Z^{2}-R^{2}, Z X\right)$ & 0 & 119 & 199 & -7 & -13 & 0 & -10 & + & - & $-(3,2)$ \\
\hline$\left(3 Z^{2}-R^{2}, X^{2}-Y^{2}\right)$ & 0 & 0 & -115 & 0 & 1 & -8 & -6 & + & + & - \\
\hline$(Z X, Z X)$ & 46 & 197 & 335 & -17 & 13 & 8 & 0 & + & + & $(2,2)$ \\
\hline$\left(Z X, X^{2}-Y^{2}\right)$ & 0 & -223 & 82 & -1 & -15 & 0 & 7 & + & - & $(2,5)$ \\
\hline$\left(X^{2}-Y^{2}, X^{2}-Y^{2}\right)$ & -34 & -56 & 93 & 0 & 17 & -28 & 4 & + & + & + \\
\hline \multicolumn{11}{|l|}{$\mathrm{FeTe}$} \\
\hline$(m, n) \backslash \boldsymbol{R}$ & {$[0,0,0]$} & {$\left[\frac{1}{2},-\frac{1}{2}, 0\right]$} & {$[1,0,0]$} & {$[1,-1,0]$} & {$\left[\frac{3}{2},-\frac{1}{2}, 0\right]$} & {$\left[0,0, \frac{c}{a}\right]$} & {$\left[\frac{1}{2},-\frac{1}{2}, \frac{c}{a}\right]$} & $\sigma_{Y}$ & $I$ & $\sigma^{L}$ \\
\hline$(X Y, X Y)$ & -452 & -378 & -11 & -41 & -1 & -31 & 12 & + & + & + \\
\hline$(X Y, Y Z)$ & 0 & 237 & 109 & 3 & -6 & 0 & -12 & + & - & $-(1,4)$ \\
\hline$\left(X Y, 3 Z^{2}-R^{2}\right)$ & 0 & -336 & 0 & 33 & -9 & 0 & 21 & - & + & + \\
\hline$(X Y, Z X)$ & 0 & 237 & 0 & 3 & 35 & 0 & -1 & - & - & $-(1,2)$ \\
\hline$\left(X Y, X^{2}-Y^{2}\right)$ & 0 & 0 & 0 & 0 & -14 & 0 & 3 & - & + & - \\
\hline$(Y Z, Y Z)$ & 44 & 156 & 103 & -15 & -12 & 13 & 37 & + & + & $(4,4)$ \\
\hline$\left(Y Z, 3 Z^{2}-R^{2}\right)$ & 0 & -122 & 0 & 17 & 6 & 0 & 11 & - & - & $-(4,3)$ \\
\hline$(Y Z, Z X)$ & 0 & 101 & 0 & -27 & -25 & 0 & 14 & - & + & $(4,2)$ \\
\hline$\left(Y Z, X^{2}-Y^{2}\right)$ & 0 & 178 & 0 & 0 & -10 & 0 & 22 & - & - & $(4,5)$ \\
\hline$\left(3 Z^{2}-R^{2}, 3 Z^{2}-R^{2}\right)$ & -480 & -73 & -53 & 3 & 8 & -67 & -23 & + & + & + \\
\hline$\left(3 Z^{2}-R^{2}, Z X\right)$ & 0 & 122 & 198 & -17 & -17 & 0 & -32 & + & - & $-(3,2)$ \\
\hline$\left(3 Z^{2}-R^{2}, X^{2}-Y^{2}\right)$ & 0 & 0 & -29 & 0 & -6 & 30 & -30 & + & + & - \\
\hline$(Z X, Z X)$ & 44 & 156 & 300 & -15 & 42 & 13 & 9 & + & + & $(2,2)$ \\
\hline$\left(Z X, X^{2}-Y^{2}\right)$ & 0 & -178 & 136 & 0 & -23 & 0 & 26 & + & - & $(2,5)$ \\
\hline$\left(X^{2}-Y^{2}, X^{2}-Y^{2}\right)$ & -211 & 66 & 52 & 9 & 12 & 16 & -24 & + & + & + \\
\hline
\end{tabular}


Table S IV. Transfer integral for the $3 d$ orbitals of the Fe sites in the FeSe and FeTe, $t_{m n}^{\mathrm{cGW}-\mathrm{SIC}}\left(R_{x}, R_{y}, R_{z}\right)$, where $t^{\mathrm{cGW}-\mathrm{SIC}}$ is the transfer integral without double-counting : $t^{\mathrm{cGW}-\mathrm{SIC}}=\left\langle\phi\left|\mathcal{H}^{\mathrm{cGW}-\mathrm{SIC}}\right| \phi\right\rangle, m$ and $n$ denote symmetries of the $3 d$ orbitals, and the axis of $\left(R_{x}, R_{y}, R_{z}\right)$ is taken along the $\mathrm{Fe}-\mathrm{Se} / \mathrm{Te}$ directions. Units are given in $\mathrm{meV}$.

\begin{tabular}{|c|c|c|c|c|c|c|c|c|c|c|}
\hline \multicolumn{11}{|l|}{ FeSe } \\
\hline$(m, n) \backslash \boldsymbol{R}$ & {$[0,0,0]$} & {$\left[\frac{1}{2},-\frac{1}{2}, 0\right]$} & {$[1,0,0]$} & {$[1,-1,0]$} & {$\left[\frac{3}{2},-\frac{1}{2}, 0\right]$} & {$\left[0,0, \frac{c}{a}\right]$} & {$\left[\frac{1}{2},-\frac{1}{2}, \frac{c}{a}\right]$} & $\sigma_{Y}$ & $I$ & $\sigma^{L}$ \\
\hline$(X Y, X Y)$ & -751 & -466 & -20 & 24 & 3 & -38 & 6 & + & + & + \\
\hline$(X Y, Y Z)$ & 0 & 201 & 106 & -26 & -3 & 0 & -9 & + & - & $-(1,4)$ \\
\hline$\left(X Y, 3 Z^{2}-R^{2}\right)$ & 0 & -391 & 0 & 27 & -3 & 0 & 8 & - & + & + \\
\hline$(X Y, Z X)$ & 0 & 201 & 0 & -26 & -9 & 0 & -4 & - & - & $-(1,2)$ \\
\hline$\left(X Y, X^{2}-Y^{2}\right)$ & 0 & 0 & 0 & 0 & 6 & 0 & -10 & - & + & - \\
\hline$(Y Z, Y Z)$ & -137 & 97 & 148 & -35 & -15 & 7 & 27 & + & + & $(4,4)$ \\
\hline$\left(Y Z, 3 Z^{2}-R^{2}\right)$ & 0 & -94 & 0 & 22 & -1 & 0 & 8 & - & - & $-(4,3)$ \\
\hline$(Y Z, Z X)$ & 0 & 199 & 0 & -37 & -36 & 0 & 16 & - & + & $(4,2)$ \\
\hline$\left(Y Z, X^{2}-Y^{2}\right)$ & 0 & 204 & 0 & -1 & 7 & 0 & 17 & - & - & $(4,5)$ \\
\hline$\left(3 Z^{2}-R^{2}, 3 Z^{2}-R^{2}\right)$ & -1075 & -67 & -76 & 5 & 11 & -4 & 3 & + & + & + \\
\hline$\left(3 Z^{2}-R^{2}, Z X\right)$ & 0 & 94 & 195 & -22 & -16 & 0 & -3 & + & - & $-(3,2)$ \\
\hline$\left(3 Z^{2}-R^{2}, X^{2}-Y^{2}\right)$ & 0 & 0 & -35 & 0 & -13 & -22 & 7 & + & + & - \\
\hline$(Z X, Z X)$ & -137 & 97 & 263 & -35 & 25 & 7 & -2 & + & + & $(2,2)$ \\
\hline$\left(Z X, X^{2}-Y^{2}\right)$ & 0 & -204 & 147 & 1 & -39 & 0 & 0 & + & - & $(2,5)$ \\
\hline$\left(X^{2}-Y^{2}, X^{2}-Y^{2}\right)$ & -299 & 186 & 19 & -20 & 8 & -35 & 17 & + & + & + \\
\hline \multicolumn{11}{|l|}{ FeTe } \\
\hline$(m, n) \backslash \boldsymbol{R}$ & {$[0,0,0]$} & {$\left[\frac{1}{2},-\frac{1}{2}, 0\right]$} & {$[1,0,0]$} & {$[1,-1,0]$} & {$\left[\frac{3}{2},-\frac{1}{2}, 0\right]$} & {$\left[0,0, \frac{c}{a}\right]$} & {$\left[\frac{1}{2},-\frac{1}{2}, \frac{c}{a}\right]$} & $\sigma_{Y}$ & $I$ & $\sigma^{L}$ \\
\hline$(X Y, X Y)$ & $\begin{array}{l}-678 \\
\end{array}$ & -410 & 52 & 5 & -2 & 23 & -13 & + & + & + \\
\hline$(X Y, Y Z)$ & 0 & 133 & 79 & -11 & -1 & 0 & 11 & + & - & $-(1,4)$ \\
\hline$\left(X Y, 3 Z^{2}-R^{2}\right)$ & 0 & -340 & 0 & 26 & -8 & 0 & 11 & - & + & + \\
\hline$(X Y, Z X)$ & 0 & 133 & 0 & -11 & -2 & -26 & 10 & - & - & $-(1,2)$ \\
\hline$\left(X Y, X^{2}-Y^{2}\right)$ & 0 & 0 & 0 & 0 & -1 & 0 & 16 & - & + & - \\
\hline$(Y Z, Y Z)$ & -146 & 42 & 120 & -28 & -17 & 11 & 15 & + & + & $(4,4)$ \\
\hline$\left(Y Z, 3 Z^{2}-R^{2}\right)$ & 0 & -88 & 0 & 24 & 6 & -23 & 14 & - & - & $-(4,3)$ \\
\hline$(Y Z, Z X)$ & 0 & 186 & 0 & -35 & -46 & 0 & 16 & - & + & $(4,2)$ \\
\hline$\left(Y Z, X^{2}-Y^{2}\right)$ & 0 & 139 & 0 & 1 & -2 & 12 & 14 & - & - & $(4,5)$ \\
\hline$\left(3 Z^{2}-R^{2}, 3 Z^{2}-R^{2}\right)$ & -974 & -114 & -114 & 21 & 26 & -1 & -1 & + & + & + \\
\hline$\left(3 Z^{2}-R^{2}, Z X\right)$ & 0 & 88 & 181 & -24 & -12 & 0 & -4 & + & - & $-(3,2)$ \\
\hline$\left(3 Z^{2}-R^{2}, X^{2}-Y^{2}\right)$ & 0 & 0 & 49 & 0 & -24 & -1 & 2 & + & + & - \\
\hline$(Z X, Z X)$ & -146 & 42 & 213 & -28 & 51 & 22 & 13 & + & + & $(2,2)$ \\
\hline$\left(Z X, X^{2}-Y^{2}\right)$ & 0 & -139 & 147 & -1 & -30 & 0 & 8 & + & - & $(2,5)$ \\
\hline$\left(X^{2}-Y^{2}, X^{2}-Y^{2}\right)$ & -495 & 292 & -47 & -9 & 20 & 5 & -13 & + & + & + \\
\hline
\end{tabular}

